\title{
Ab Initio Study of Strain Effects on the Quasiparticle Bands and Effective Masses in Silicon
}

\author{
Mohammed Bouhassoune ${ }^{1}$ and Arno Schindlmayr ${ }^{2}$ \\ ${ }^{1}$ Peter Grünberg Institut and Institute for Advanced Simulation, Forschungszentrum Jülich and JARA, 52425 Jülich, Germany \\ ${ }^{2}$ Department Physik, Universität Paderborn, 33095 Paderborn, Germany
}

Correspondence should be addressed to Arno Schindlmayr; arno.schindlmayr@uni-paderborn.de

Received 1 December 2014; Accepted 16 January 2015

Academic Editor: Da-Ren Hang

Copyright ( 2015 M. Bouhassoune and A. Schindlmayr. This is an open access article distributed under the Creative Commons Attribution License, which permits unrestricted use, distribution, and reproduction in any medium, provided the original work is properly cited.

\begin{abstract}
Using ab initio computational methods, we study the structural and electronic properties of strained silicon, which has emerged as a promising technology to improve the performance of silicon-based metal-oxide-semiconductor field-effect transistors. In particular, higher electron mobilities are observed in $n$-doped samples with monoclinic strain along the [110] direction, and experimental evidence relates this to changes in the effective mass as well as the scattering rates. To assess the relative importance of these two factors, we combine density-functional theory in the local-density approximation with the $G W$ approximation for the electronic self-energy and investigate the effect of uniaxial and biaxial strains along the [110] direction on the structural and electronic properties of Si. Longitudinal and transverse components of the electron effective mass as a function of the strain are derived from fits to the quasiparticle band structure and a diagonalization of the full effective-mass tensor. The changes in the effective masses and the energy splitting of the conduction-band valleys for uniaxial and biaxial strains as well as their impact on the electron mobility are analyzed. The self-energy corrections within $G W$ lead to band gaps in excellent agreement with experimental measurements and slightly larger effective masses than in the local-density approximation.
\end{abstract}

\section{Introduction}

Silicon retains its place as the most prominent material used in technological applications such as metaloxide-semiconductor field-effect transistor (MOSFET) based devices. This success stems from the sustained increase in performance that could be achieved, over many years, by continuous geometric downscaling. Nowadays, however, as devices approach their physical and geometrical limits, other solutions must be found to uphold the trend of Moore's law. Strain engineering has become one of the promising scaling vectors in this context, because it can lead to higher carrier mobility and faster switching times than in conventional devices based on unstrained silicon while remaining fully compatible with existing manufacturing technology $[1,2]$. Strained silicon is already used in massscale industrial production since the $90 \mathrm{~nm}$ node, together with new dielectric materials and other boost factors. The most established method to obtain strained layers of silicon is to grow a silicon-germanium film on top of a standard wafer, which then serves as an atomic template for the subsequent epitaxial growth of silicon layers [3]. These adopt the larger lateral lattice constant of the silicon-germanium substrate and therefore exhibit biaxial tensile strain. With this growth process, around $1 \%$ strain in the Si layer can be achieved, which enhances the electron mobility by a factor of 1.8-2.0 [2, 4-6]. The enhancement of the hole mobility is negligible [6], on the other hand, as this would require significantly higher tensile strain, which can not easily be realized by epitaxy. Different techniques have been pursued in this situation until uniaxial strain was introduced into the MOSFET channel [710], which promises to enhance the electron and hole mobility even at smaller strain than $1 \%[10,11]$. In order to implement uniaxial strain, stress liner techniques are commonly used, where capping layers, usually of silicon nitride, are grown on top of the transistor to produce compressive or tensile strain depending on the deposition conditions. Heteroepitaxial strain techniques, where epitaxial growth of SiGe and $\mathrm{SiC}$ in etched recesses near the source and drain generates uniaxial strain due to the lattice mismatch with the silicon 
channel, may also be employed. In this way, a hole mobility enhancement by a factor of 2 has been observed for $p$-type MOSFETs with $2 \mathrm{GPa}$ uniaxial stress [12, 13].

Although early theoretical studies [14-18] already provided a qualitative understanding of strain effects on the electronic structure of $\mathrm{Si}$, actual progress was so far mainly driven by experiments that measure the impact of a particular strain configuration on the charge carrier mobility, for instance, through changes in the resistivity. Several parameters, in fact, independently influence the carrier mobility in MOSFETs: first, the effective masses are inversely proportional to the curvature of the energy bands. If strain modifies the energy bands, this will lead to changes in the effective masses. Second, the scattering rates of the charge carriers can be reduced by a partial lifting of band-edge degeneracies in strained samples with reduced symmetry. Indeed, for certain strain configurations, the number of available scattering channels for electrons residing in the lowest conduction-band valleys naturally decreases. In addition, surface roughness and other process-induced factors may influence the mobility in actual devices. Therefore, it is difficult to assess the quantitative role of the individual contributing factors on the basis of experimental measurements alone. To answer this question, we conducted a combined theoretical and experimental study of biaxial tensile strain in the (001) plane of $n$-type Si [19], corresponding to a tetragonal distortion of the unit cell. Our measurements indicate no change in the electron effective mass, obtained from the temperature dependence of the Shubnikov-de Haas oscillation amplitudes, which is consistent with our ab initio band-structure calculations that show no warping of the lowest conduction band up to $1 \%$ applied strain. The observed enhancement of the mobility is hence attributed mainly to the partial splitting of the originally sixfold degenerate conduction-band valleys, which reduces the scattering rate. In the case of biaxial tensile strain in the (111) plane, corresponding to a trigonal distortion of the unit cell, the six conduction-band valleys remain degenerate, so that the scattering channels are unaffected. As all components of the effective mass tensor furthermore increase with strain [20], the trigonal distortion may even reduce the electron mobility. On the other hand, for monoclinic deformations along the [110] direction, experimental evidence suggests that both the effective mass of the charge carriers and the scattering rate contribute to the observed high electron mobility in $n$-doped samples. It was also reported that uniaxial strain induces a much larger mobility enhancement for both electrons and holes [21-23] than wafer-based biaxial strain [24].

Theoretical simulations of the carrier mobility require an accurate knowledge of the electronic band structure under strain, but the vast majority of band-structure calculations dealing with strained Si rely on empirical approaches that require input parameters from experiments or, like densityfunctional theory (DFT) within the local-density approximation (LDA), exhibit systematic errors. To circumvent these problems, here we instead employ ab initio computational methods that combine DFT-LDA and the $G W$ approximation for the electronic self-energy in order to obtain highly accurate quantitative results for the band structure, the variation of the effective masses, and the valley splitting as functions of the applied strain. We focus on uniaxial and biaxial [110] strains, because this monoclinic deformation is the most promising for achieving high charge carrier mobilities.

This paper is organized as follows. In Section 2 we give an overview of our computational method and discuss the structural parameters of silicon under a monoclinic deformation along the [110] direction. In Section 3 we then present our results for the electronic structure and compare the effects of tensile biaxial and uniaxial strains on the electron mobility by analyzing the changes in the effective masses and the splitting of the conduction-band valleys. We finally summarize our conclusions in Section 4.

\section{Computational Method}

We start by performing a series of $a b$ initio calculations using density-functional theory [25] within the local-density approximation [26] to determine the structural properties of silicon under uniaxial and biaxial strains in the [110] direction. While the ground-state total energy for a given atomic configuration is accurately described by the DFTLDA approach, the Kohn-Sham eigenvalue dispersion differs systematically from the true quasiparticle band structure. In particular, band gaps are severely underestimated, but the band curvature and hence the effective masses also show deviations. For this reason, we employ many-body perturbation theory and the $G W$ approximation for the electronic self-energy [27], which yields quasiparticle band structures in excellent agreement with experimental photoemission data. The derived effective masses also agree well with experimentally measured values, not only for solids like silicon [19], but also for very different materials, such as organic semiconductors $[28,29]$.

Our calculations use nonlocal norm-conserving pseudopotentials and a plane-wave basis set with a cutoff energy of 20 Ry. We choose the parametrization of Perdew and Zunger [30] for the exchange-correlation functional. The structural optimization within DFT-LDA is performed with a $10 \times 10 \times$ $10 \mathbf{k}$-point set in the full Brillouin zone, whereas a sampling of $4 \times 4 \times 4 \mathbf{k}$ points suffices for the quasiparticle shifts in the $G W$ approximation. The self-energy is constructed with 100 unoccupied bands in the standard perturbative way from the Kohn-Sham orbitals, and we evaluate the dynamic screening function in the random-phase approximation without recourse to plasmon-pole models [31, 32].

Crystal deformations are described by the strain tensor $\varepsilon$, which transforms the primitive lattice vectors $\mathbf{a}_{1}, \mathbf{a}_{2}$, and $\mathbf{a}_{3}$ according to $\mathbf{a}_{i}^{\prime}=(I+\varepsilon) \mathbf{a}_{i}$, where $I$ denotes the identity matrix. For monoclinic deformations along the [110] direction, the strain tensor takes the following form:

$$
\varepsilon=\left(\begin{array}{ccc}
\frac{\varepsilon_{\|}+\varepsilon_{\perp}}{2} & \frac{\varepsilon_{\|}-\varepsilon_{\perp}}{2} & 0 \\
\frac{\varepsilon_{\|}-\varepsilon_{\perp}}{2} & \frac{\varepsilon_{\|}+\varepsilon_{\perp}}{2} & 0 \\
0 & 0 & \varepsilon_{\|}
\end{array}\right),
$$

where $\varepsilon_{\|}$is the strain component in the (110) plane and $\varepsilon_{\perp}$ is the out-of-plane strain parameter along the [110] 


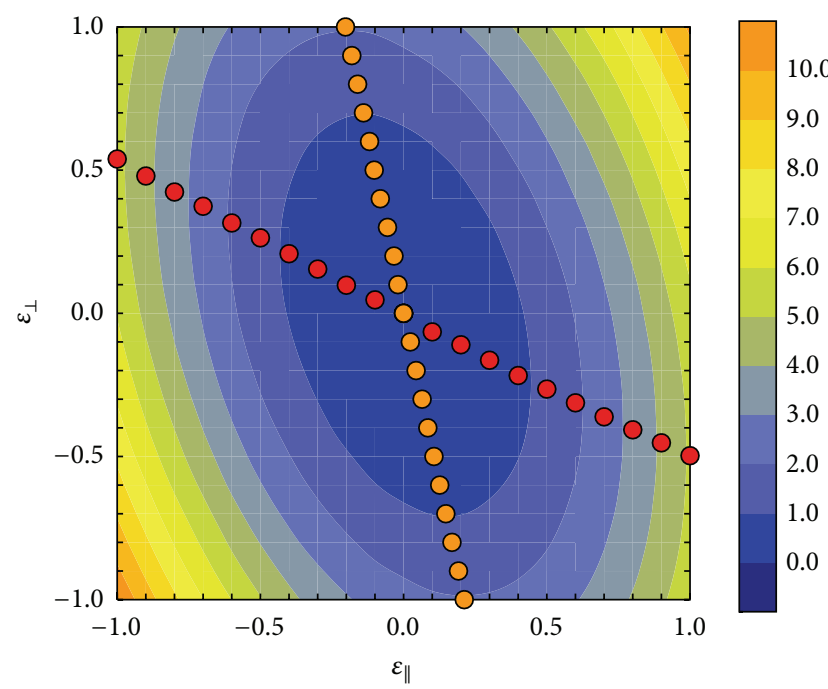

FIgure 1: Contour plot of the total energy in $\mathrm{meV}$ per unit cell as a function of the in-plane strain parameter $\varepsilon_{\|}$and the out-of-plane parameter $\varepsilon_{\perp}$ for monoclinic deformations along the [110] direction. The orange and red dots refer to configurations with uniaxial and biaxial strains, respectively.

direction. Under equilibrium conditions silicon crystallizes in the diamond structure with two atoms per unit cell and 24 symmorphic point symmetries. The monoclinic distortion reduces the number of symmetry operations to four. As a consequence, the relative positions of the two atoms inside the unit cell are no longer fixed by symmetry constraints, and internal displacements will occur. In our calculations we take this internal relaxation fully into account and determine the optimal sublattice spacing by minimizing the total energy separately for each configuration. The internal displacement parameter $\zeta$ derived from our results depends linearly on the strain, but the deviation from the limiting value $\zeta=$ 0.53 at zero strain is small for experimentally achievable deformations.

The elastic moduli as well as related quantities can be determined from the change in total energy as a function of the applied strain. A contour plot of the total energy $E\left(\varepsilon_{\|}, \varepsilon_{\perp}\right)$ for monoclinic deformations is shown in Figure 1. To simulate uniaxial (orange dots) and biaxial (red dots) strains, we fix one of the two strain parameters and determine the other by a constrained energy minimization:

$$
\frac{\partial E\left(\varepsilon_{\|}, \varepsilon_{\perp}=\varepsilon_{\text {uniax }}\right)}{\partial \varepsilon_{\|}}=0, \quad \frac{\partial E\left(\varepsilon_{\|}=\varepsilon_{\text {biax }}, \varepsilon_{\perp}\right)}{\partial \varepsilon_{\perp}}=0,
$$

respectively. For small deformations the relation between the two components of the strain is linear and is given by the Poisson ratio $v=-\varepsilon_{\perp} / \varepsilon_{\|}$. The values for biaxial and uniaxial strains obtained here directly from first principles are consistent with those derived from the elastic constants calculated in [20] and with experiments.

\section{Results and Discussion}

As an illustration, Figure 2 shows the quasiparticle band structure for unstrained silicon and for uniaxial strain $\varepsilon_{\text {uniax }}=$ $2.5 \%$ along the [110] direction. The $G W$ approximation yields a fundamental band gap of $1.17 \mathrm{eV}$ for bulk silicon, which matches the experimental value [33], while the DFT-LDA eigenvalue gap is only $0.46 \mathrm{eV}$ due to the well-known systematic underestimation resulting from the local approximation of the exchange-correlation functional. For tensile uniaxial strain the sixfold degenerate conduction-band minimum splits into two valleys $\Delta_{2}$ with lower energy along the $\Gamma-Z$ direction and four others $\Delta_{4}$ with higher energy along the $\Gamma-X$ direction. The situation is different for tensile biaxial strain, where the $\Delta_{2}$ valleys are higher in energy than the $\Delta_{4}$ valleys. In both cases, as displayed in Figure 3, the band gap decreases with strain, but the splitting between the $\Delta_{2}$ and $\Delta_{4}$ valleys is more pronounced for uniaxial strain. Furthermore, for uniaxial strain the splitting grows with increasing strain, which reduces the scattering rate because only the two lower occupied $\Delta_{2}$ valleys affect the electron mobility. In the case of biaxial strain, the splitting between the $\Delta_{4}$ and $\Delta_{2}$ valleys is much smaller than that for uniaxial strain, and the energetic ordering in fact changes for strain above $\varepsilon_{\text {biax }}=3.25 \%$, where the $\Delta_{2}$ valleys are lower in energy. This regime of large tensile biaxial strain in the (110) plane might hence be interesting for an enhancement of the electron mobility. The crossing of the valley positions was already observed in an earlier empirical tight-binding study of the band-edge energies of strained silicon grown coherently on a $\mathrm{Si}_{1-x} \mathrm{Ge}_{x}(110)$ substrate [34], but the crossing occurred at a smaller germanium concentration of $x=0.5$ than that corresponding to the strain value $3.25 \%$ predicted here by our $a b$ initio calculation, which includes no empirical input parameters.

As mentioned in the introduction, the scattering rate and the effective mass are two principal factors that affect the charge carrier mobility. The splitting of the bands reduces the scattering rate if it exceeds the thermal energy, and the warping of the bands has an impact on the effective masses, which are related to the band curvature. This point can be seen in Figure 4, where we show contour plots of the quasiparticle energy $\epsilon_{n \mathbf{k}}$ in the $k_{z}=0$ plane of the Brillouin zone for the lowest conduction band of silicon without strain and with 3.0\% uniaxial strain. The figure clearly illustrates the symmetry reduction due to the applied uniaxial strain in the lower panel, as the [110] and [1 10$]$ directions are no longer equivalent for a monoclinic distortion of the unit cell.

To obtain the effective masses as a function of the strain, we must construct the components of the full tensor and then diagonalize it for each band-edge state. Due to the symmetry reduction resulting from the monoclinic distortion, which also affects the geometry of the Brillouin zone, the fourfold degenerate $\Delta_{4}$ valleys of the conduction band are no longer found along the [100] and [010] axes, as for unstrained silicon, but move away from these high-symmetry lines into the $k_{z}=0$ plane. This situation is illustrated for uniaxial strain in the lower panel of Figure 4, where all valleys are seen to lie in the second and fourth quadrant. As a first 

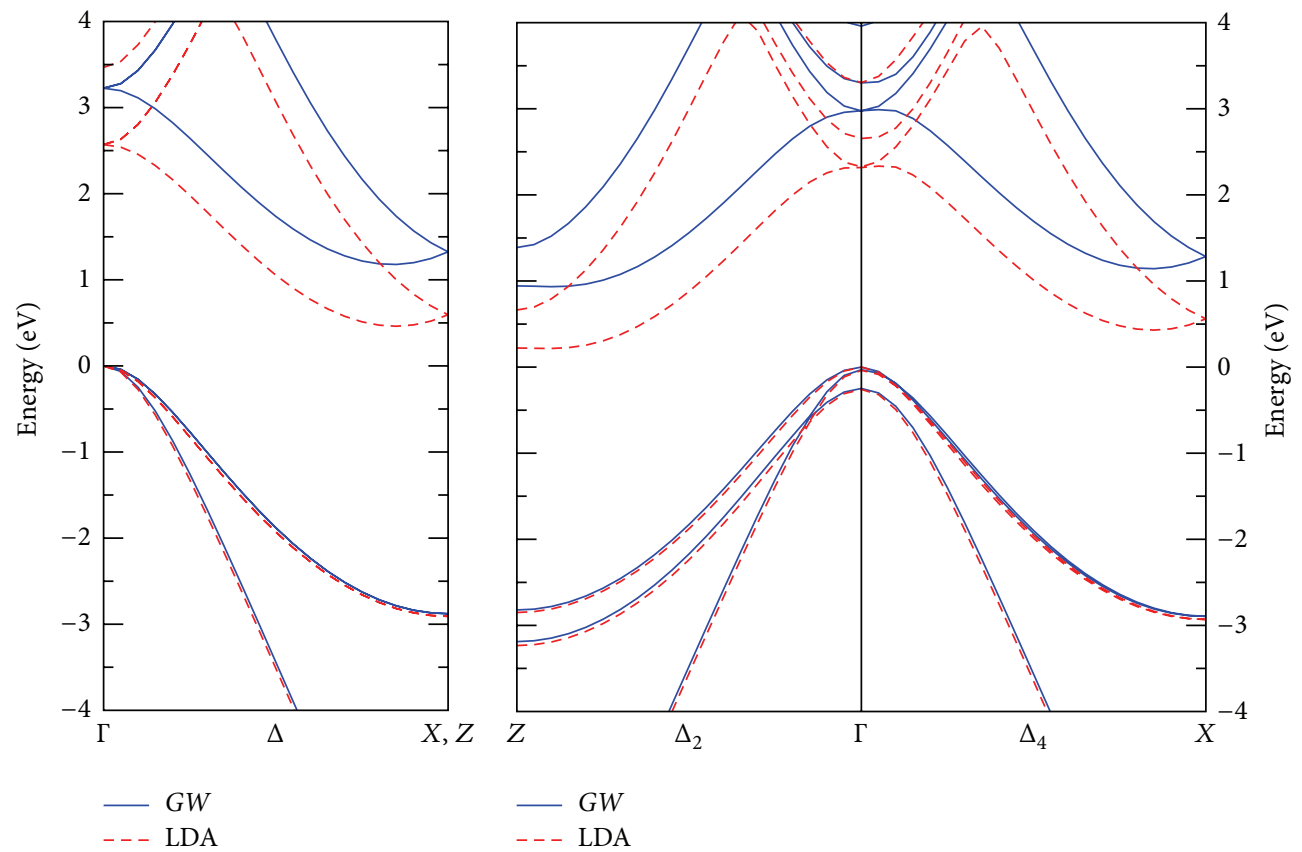

FIgURE 2: Band structure of Si within DFT-LDA (dashed lines) and GW (solid lines) without strain (left) and with $2.5 \%$ uniaxial strain in the [110] direction (right). The six originally degenerate conduction-band minima (left panel) split into two lower $\Delta_{2}$ valleys along the $\Gamma-Z$ direction and four higher $\Delta_{4}$ valleys along the $\Gamma-X$ direction (right panel).

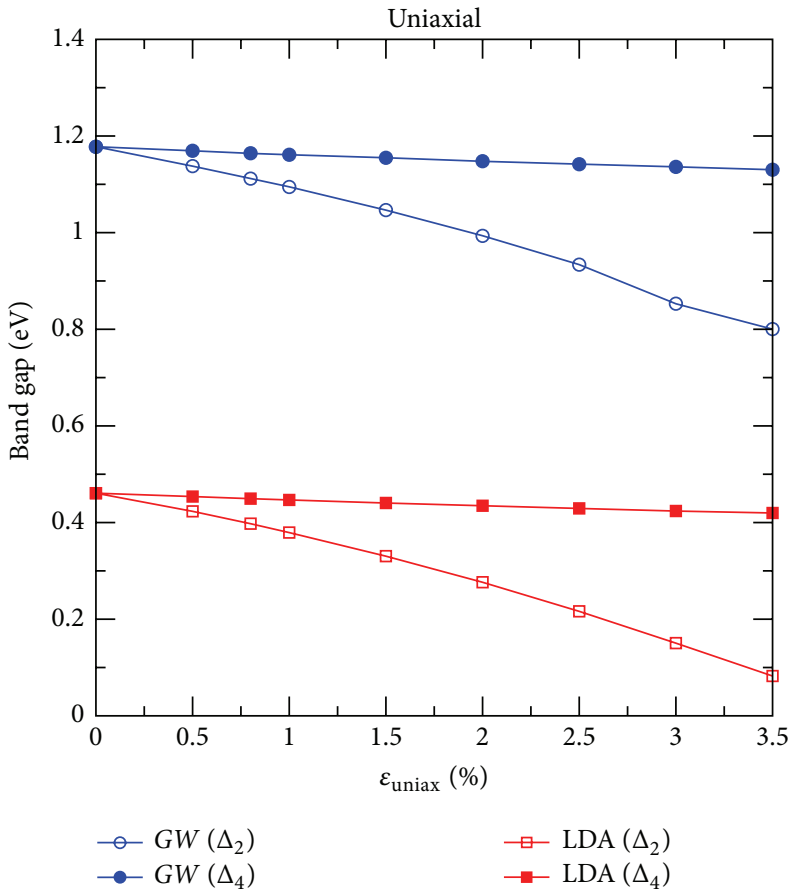

(a)

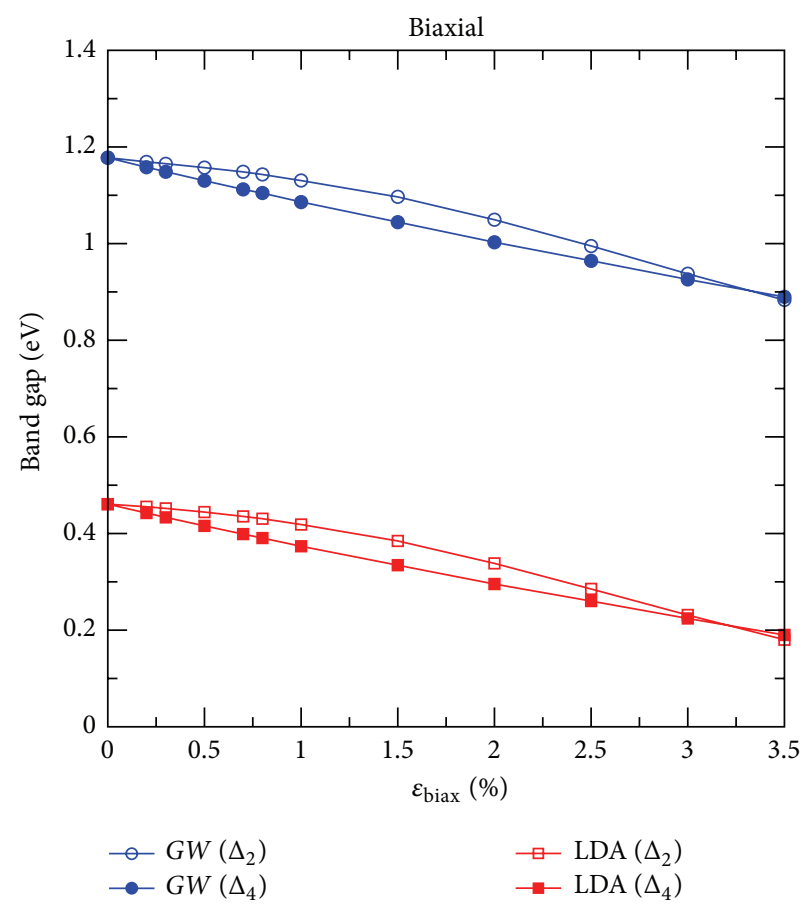

(b)

FIGURE 3: Band gap of silicon versus strain for tensile uniaxial (a) and biaxial (b) strains along the [110] direction within DFT-LDA and GW for both the $\Delta_{2}$ and $\Delta_{4}$ valleys. 


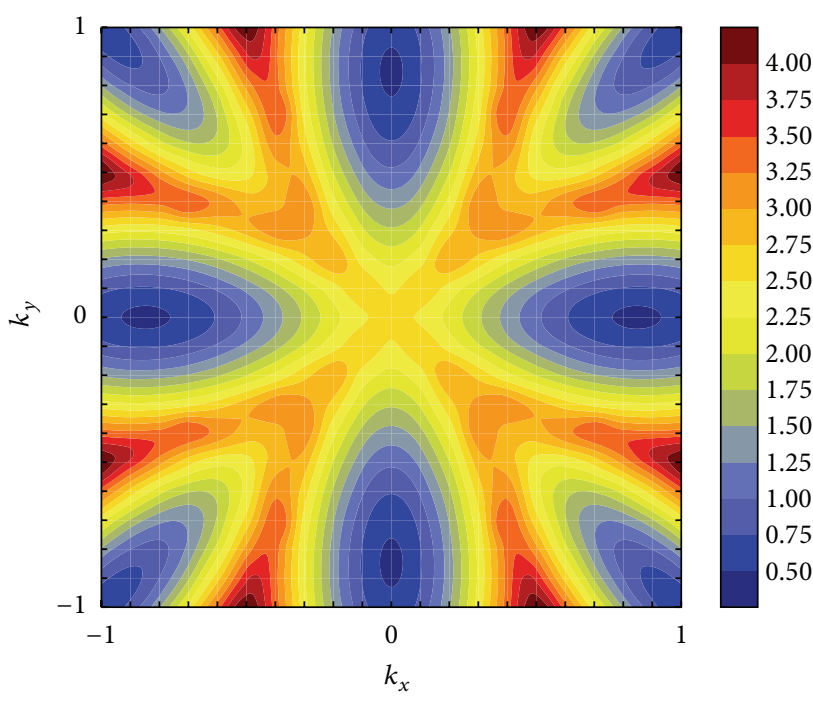

(a)

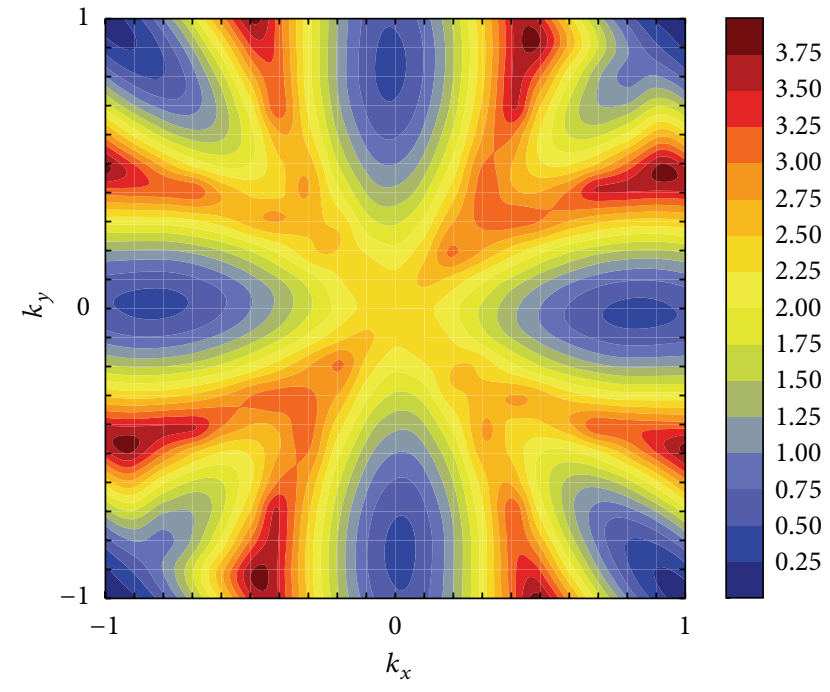

(b)

FIGURE 4: Contour plot of $\epsilon_{n \mathbf{k}}$ in the $k_{z}=0$ plane of the Brillouin zone for the lowest conduction band without strain (a) and with $3.0 \%$ uniaxial strain in the [110] direction (b). The quasiparticle energies are given in $\mathrm{eV}$ and relative to the minimum of the band in each configuration.

step, we determine the position $\mathbf{k}_{0}$ of a band minimum in the $k_{z}=0$ plane using a two-dimensional steepest descent method. Subsequently, we calculate the curvatures $\partial^{2} \epsilon_{n \mathbf{k}} / \partial k_{\alpha}^{2}$ with $\alpha \in\{x, y, z\}$ at the band minimum from polynomial fits on a dense mesh of $\mathbf{k}$ points along the three Cartesian axes as well as $\partial^{2} \epsilon_{n \mathbf{k}(\lambda)} / \partial \lambda^{2}$ along a diagonal path in the [110] direction parametrized by $\mathbf{k}(\lambda)=\mathbf{k}_{0}+\lambda(1,1,0) / \sqrt{2}$. The offdiagonal components of the effective-mass tensor

$$
\frac{1}{m^{*}}=\left(\begin{array}{ccc}
\frac{\partial^{2} \epsilon_{n \mathbf{k}}}{\partial k_{x}^{2}} & \frac{\partial^{2} \epsilon_{n \mathbf{k}}}{\partial k_{x} \partial k_{y}} & 0 \\
\frac{\partial^{2} \epsilon_{n \mathbf{k}}}{\partial k_{x} \partial k_{y}} & \frac{\partial^{2} \epsilon_{n \mathbf{k}}}{\partial k_{y}^{2}} & 0 \\
0 & 0 & \frac{\partial^{2} \epsilon_{n \mathbf{k}}}{\partial k_{z}^{2}}
\end{array}\right)
$$

at $\mathbf{k}_{0}$ can then be constructed according to

$$
\frac{\partial^{2} \epsilon_{n \mathbf{k}}}{\partial k_{x} \partial k_{y}}=\frac{\partial^{2} \epsilon_{n \mathbf{k}(\lambda)}}{\partial \lambda^{2}}-\frac{1}{2}\left(\frac{\partial^{2} \epsilon_{n \mathbf{k}}}{\partial k_{x}^{2}}+\frac{\partial^{2} \epsilon_{n \mathbf{k}}}{\partial k_{y}^{2}}\right)
$$

The diagonalization of this tensor yields three distinct effective-mass values, of which one is large and can be identified with the longitudinal effective mass $m_{l}^{*}$ and two are transverse masses with smaller numerical values $m_{t}^{*}$. To avoid the laborious minimum search by the steepest descent method, we have also attempted, as an approximation, to evaluate the band curvatures at the $\mathbf{k}$ point obtained by applying the strain tensor to the location $(0.84) X$ of the conduction-band minimum of unstrained silicon, which is very close to $\mathbf{k}_{0}$. As the resulting values for the effective masses are almost identical, most of our data are actually obtained in this way. The reduced symmetry due to the monoclinic distortion does not shift the $\Delta_{2}$ valleys away from the [001] axis. Therefore, no steepest descent method is required, and the minima are easily found by a onedimensional search along the $\Gamma-Z$ direction. Furthermore, the effective-mass tensor is diagonal in this case with one longitudinal and two degenerate transverse components.

In Figures 5 and 6 we display the calculated variation of the longitudinal and transverse components of the electron effective mass for uniaxial and biaxial strains, respectively. The splitting of the conduction band into $\Delta_{2}$ and $\Delta_{4}$ valleys implies that these have distinct effective masses. The effective masses associated with the $\Delta_{4}$ valleys are almost unaffected by the distortion and remain close to those of unstrained silicon. As already stated above, the fourfold degenerate $\Delta_{4}$ valleys are higher in energy for uniaxial strain and lower in energy for biaxial strain up to $\varepsilon_{\text {biax }}=3.25 \%$. Tensile uniaxial strain is hence of particular interest, because the twofold degenerate $\Delta_{2}$ valleys not only are the lowest in energy but also feature small transverse electron effective masses $m_{t[110]}$ that decrease even further as $\varepsilon_{\text {uniax }}$ increases. In the case of biaxial strain, the $\Delta_{2}$ valleys show a similar behavior as a function of $\varepsilon_{\text {biax }}$, but they are higher in energy than the $\Delta_{4}$ valleys and thus irrelevant for electron transport. According to our quantitative assessment of the band structure, the splitting between the $\Delta_{4}$ and $\Delta_{2}$ valleys exceeds the thermal energy $k_{B} T=25 \mathrm{meV}$ at room temperature, so that only the $\Delta_{4}$ valleys will be populated until the strain exceeds $3.25 \%$, where the ordering of the $\Delta_{4}$ and $\Delta_{2}$ valleys changes. Figures 5 and 6 further show that the longitudinal effective mass associated with the $\Delta_{2}$ valleys grows rapidly with the strain strength for both uniaxial and biaxial strains.

A comparison between the DFT-LDA and GW results reveals that the latter systematically predicts slightly larger values for the effective masses. The narrowing of the energy dispersion in the $G W$ approximation, which gives rise to the larger effective masses, has been observed in other 


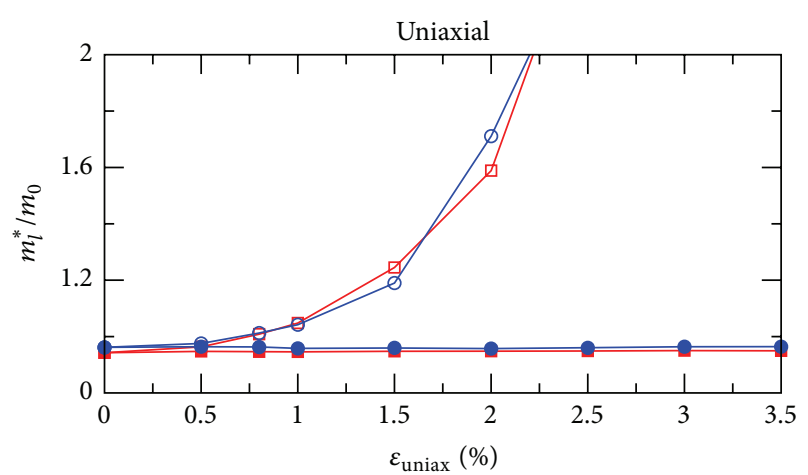

(a)

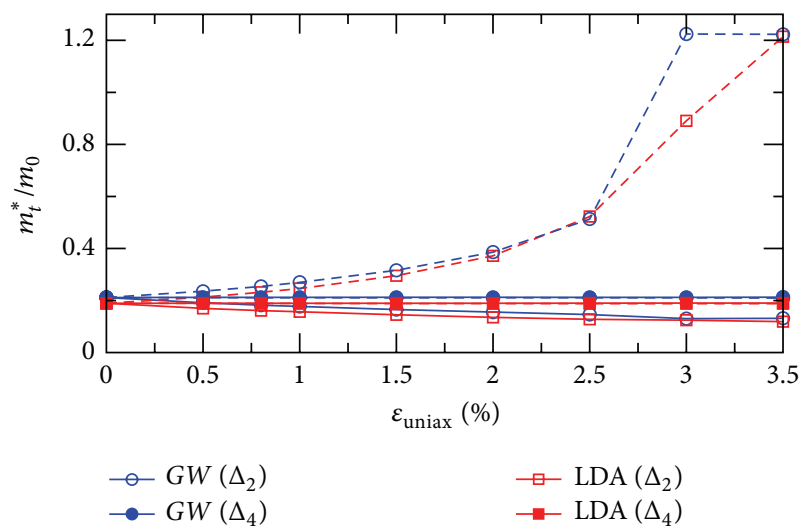

(b)

Figure 5: Longitudinal (a) and transverse (b) components of the electron effective mass in silicon as a function of tensile uniaxial strain in the [110] direction. The solid and dashed lines distinguish the two transverse elements of the mass tensor.

systems before. It stems from the interaction of the electron with its environment, which rearranges due to quantummechanical correlation and acts back on the electron, slowing its further motion. Mathematically, the self-energy insertion redistributes spectral weight from the density of states to create satellite resonances [35].

\section{Conclusions}

We have studied the structural deformation of bulk silicon for uniaxial and biaxial strains along the [110] direction with full internal and volume relaxation using DFT-LDA. On this basis, we then quantitatively determined the electronic structure of strained silicon with proper quasiparticle corrections using the $G W$ approximation for the electronic self-energy. For tensile uniaxial strain with realistic strain parameters, we find that the splitting between the occupied $\Delta_{2}$ and the unoccupied $\Delta_{4}$ valleys is large, implying a reduced intervalley scattering, and the electron mobility is further enhanced by a reduced transverse effective mass. No such reduction is found for the fourfold degenerate occupied $\Delta_{4}$ valleys in the case of biaxial strain. In previous work we also examined the effect of strain along other directions and demonstrated that the electron effective mass is not modified by biaxial

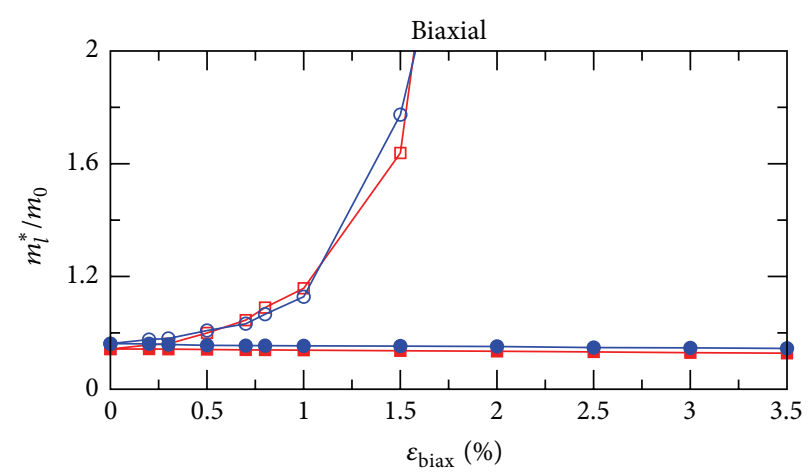

(a)

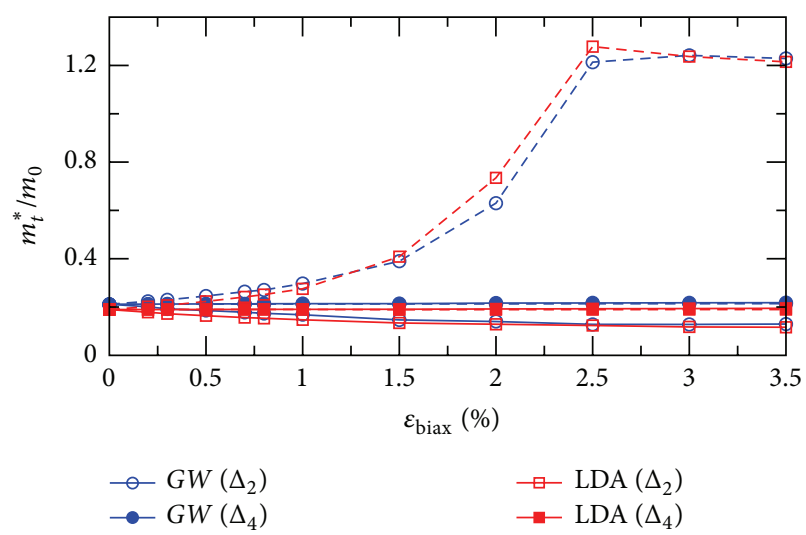

(b)

Figure 6: Longitudinal (a) and transverse (b) components of the electron effective mass in silicon as a function of tensile biaxial strain in the (110) plane. The solid and dashed lines distinguish the two transverse elements of the mass tensor.

strain along the [001] direction [19]. Therefore, any increase of the mobility stems entirely from the reduced scattering rate due to the splitting of the conduction-band valleys in this case. Biaxial trigonal distortions along the [111] direction are also not relevant for increasing the mobility [20]. We hence conclude that tensile uniaxial strain along the [110] direction is indeed the most promising configuration to achieve high electron mobility in silicon, as both the scattering rate and the effective mass are reduced in this scenario. Tensile biaxial strain in the (110) plane could also be interesting, but a large strain value above $3.25 \%$ is then needed, which might be difficult to realize in practical epitaxy.

\section{Conflict of Interests}

The authors declare that there is no conflict of interests regarding the publication of this paper.

\section{Acknowledgments}

This work was funded in part by the EU's Seventh Framework Programme through the European Theoretical Spectroscopy Facility e-I3 (INFRA-2007-211956). Mohammed 
Bouhassoune further acknowledges support from the HGFYIG programme Funsilab-Functional Nanoscale Structure Probe and Simulation Laboratory (VH-NG-717).

\section{References}

[1] S. E. Thompson, M. Armstrong, C. Auth et al., "A $90 \mathrm{~nm}$ logic technology featuring strained-silicon," IEEE Transactions on Electron Devices, vol. 51, no. 11, pp. 1790-1797, 2004.

[2] M. T. Currie, C. W. Leitz, T. A. Langdo, G. Taraschi, E. A. Fitzgerald, and D. A. Antoniadis, "Carrier mobilities and process stability of strained $\mathrm{Si} \mathrm{n-} \mathrm{and} \mathrm{p-MOSFETs} \mathrm{on} \mathrm{SiGe}$ virtual substrates," Journal of Vacuum Science and Technology B: Microelectronics and Nanometer Structures, vol. 19, no. 6, pp. 2268-2279, 2001.

[3] E. A. Fitzgerald, Y.-H. Xie, M. L. Green et al., "Totally relaxed $\mathrm{Ge}_{x} \mathrm{Si}_{1-x}$ layers with low threading dislocation densities grown on Si substrates," Applied Physics Letters, vol. 59, no. 7, pp. 811813, 1991.

[4] J. Welser, J. L. Hoyt, and J. F. Gibbons, "Electron mobility enhancement in strained-Si N-type metal-oxide-semiconductor field-effect transistors," IEEE Electron Device Letters, vol. 15, no. 3, pp. 100-102, 1994.

[5] C. K. Maiti, L. K. Bera, and S. Chattopadhyay, "Strained-Si heterostructure field effect transistors," Semiconductor Science and Technology, vol. 13, no. 11, pp. 1225-1246, 1998.

[6] K. Rim, J. Chu, H. Chen et al., "Characteristics and device design of sub-100 nm strained Si N- and PMOSFETs," in Proceedings of the Symposium on VLSI Technology. Digest of Technical Papers, pp. 98-99, June 2002.

[7] S. Ito, H. Namba, K. Yamaguchi et al., "Mechanical stress effect of etch-stop nitride and its impact on deep submicron transistor design," in Proceedings of the IEEE International Electron Devices Meeting (IEDM '00). Technical Digest, pp. 247250, San Francisco, Calif, USA, December 2000.

[8] T. Ghani, M. Amstrong, C. Auth et al., "A $90 \mathrm{~nm}$ high volume manufacturing logic technology featuring novel $45 \mathrm{~nm}$ gate length strained silicon CMOS transistors," in Proceedings of the IEEE International Electron Devices Meeting (IEDM '03). Technical Digest, pp. 978-980, Washington, DC, USA, December 2003.

[9] K. Uchida, R. Zednik, C.-H. Lu et al., "Experimental study of biaxial and uniaxial strain effects on carrier mobility in bulk and ultrathin-body SOI MOSFETs," in Proceedings of the IEEE International Electron Devices Meeting (IEDM '04). Technical Digest, pp. 229-232, December 2004.

[10] S. E. Thompson, M. Armstrong, C. Auth et al., "A logic nanotechnology featuring strained-silicon," IEEE Electron Device Letters, vol. 25, no. 4, pp. 191-193, 2004.

[11] B. M. Haugerud, L. A. Bosworth, and R. E. Belford, "Mechanically induced strain enhancement of metal-oxidesemiconductor field effect transistors," Journal of Applied Physics, vol. 94, no. 6, pp. 4102-4107, 2003.

[12] L. Smith, V. Moroz, G. Eneman et al., "Exploring the limits of stress-enhanced hole mobility," IEEE Electron Device Letters, vol. 26, no. 9, pp. 652-654, 2005.

[13] L. Washington, F. Nouri, S. Thirupapuliyur et al., "pMOSFET with $200 \%$ mobility enhancement induced by multiple stressors," IEEE Electron Device Letters, vol. 27, no. 6, pp. 511-513, 2006.

[14] Q. M. Ma, K. L. Wang, and J. N. Schulman, "Band structure and symmetry analysis of coherently grown $\mathrm{Si}_{1-x} \mathrm{Ge}_{x}$ alloys on oriented substrates," Physical Review B, vol. 47, no. 4, pp. 1936$1953,1993$.

[15] C. Tserbak, H. M. Polatoglou, and G. Theodorou, "Unified approach to the electronic structure of strained Si/Ge superlattices," Physical Review B, vol. 47, no. 12, pp. 7104-7124, 1993.

[16] M. Gell, "Effective masses and sum rules in strained $\mathrm{Si} / \mathrm{Ge}$ structures," Physical Review B, vol. 41, no. 11, pp. 7611-7614, 1990.

[17] M. M. Rieger and P. Vogl, "Electronic-band parameters in strained $\mathrm{Si}_{1-x} \mathrm{Ge}_{x}$ alloys on $\mathrm{Si}_{1-y} \mathrm{Ge}_{y}$ substrates," Physical Review B, vol. 48, no. 19, pp. 14276-14287, 1993.

[18] M. V. Fischetti and S. E. Laux, "Band structure, deformation potentials, and carrier mobility in strained $\mathrm{Si} \mathrm{Ge}$, and SiGe alloys," Journal of Applied Physics, vol. 80, no. 4, pp. 2234-2252, 1996.

[19] S. F. Feste, T. Schäpers, D. Buca et al., "Measurement of effective electron mass in biaxial tensile strained silicon on insulator," Applied Physics Letters, vol. 95, no. 18, Article ID 182101, 2009.

[20] M. Bouhassoune and A. Schindlmayr, "Electronic structure and effective masses in strained silicon," Physica Status Solidi $C$, vol. 7, no. 2, pp. 460-463, 2010.

[21] K. Uchida, T. Krishnamohan, K. C. Saraswat, and Y. Nishi, "Physical mechanisms of electron mobility enhancement in uniaxial stressed MOSFETs and impact of uniaxial stress engineering in ballistic regime," in Proceedings of the IEEE International Electron Devices Meeting (IEDM '05). Technical Digest, pp. 129-132, Washington, DC, USA, December 2005.

[22] S. E. Thompson, G. Sun, Y. S. Choi, and T. Nishida, "Uniaxialprocess-induced strained-Si: extending the CMOS roadmap," IEEE Transactions on Electron Devices, vol. 53, no. 5, pp. 10101020, 2006.

[23] F. Rochette, M. Cassé, M. Mouis et al., "Experimental evidence and extraction of the electron mass variation in [110] uniaxially strained MOSFETs," Solid-State Electronics, vol. 51, no. 11-12, pp. 1458-1465, 2007.

[24] J. L. Hoyt, H. M. Nayfeh, S. Eguchi et al., "Strained silicon MOSFET technology," in Proceedings of the IEEE International Electron Devices Meeting (IEDM '02). Technical Digest, pp. 2326, December 2002.

[25] P. Hohenberg and W. Kohn, "Inhomogeneous electron gas," Physical Review, vol. 136, no. 3B, pp. B864-B871, 1964.

[26] W. Kohn and L. J. Sham, "Self-consistent equations including exchange and correlation effects," Physical Review, vol. 140, no. 4A, pp. A1133-A1138, 1965.

[27] L. Hedin, "New method for calculating the one-particle Green's function with application to the electron-gas problem," Physical Review, vol. 139, no. 3A, pp. A796-A823, 1965.

[28] S. Yanagisawa, Y. Morikawa, and A. Schindlmayr, "HOMO band dispersion of crystalline rubrene: effects of self-energy corrections within the GW approximation," Physical Review B, vol. 88, no. 11, Article ID 115438, 2013.

[29] S. Yanagisawa, Y. Morikawa, and A. Schindlmayr, "Theoretical investigation of the band structure of picene single crystals within the GW approximation," Japanese Journal of Applied Physics, vol. 53, no. 5S1, Article ID 05FY02, 2014.

[30] J. P. Perdew and A. Zunger, "Self-interaction correction to density-functional approximations for many-electron systems," Physical Review B, vol. 23, no. 10, pp. 5048-5079, 1981.

[31] M. M. Rieger, L. Steinbeck, I. D. White, H. N. Rojas, and R. W. Godby, "The $G W$ space-time method for the self-energy of large systems," Computer Physics Communications, vol. 117, no. 3, pp. 211-228, 1999. 
[32] C. Freysoldt, P. Eggert, P. Rinke, A. Schindlmayr, R. W. Godby, and M. Scheffler, "Dielectric anisotropy in the $G W$ space-time method," Computer Physics Communications, vol. 176, no. 1, pp. 1-13, 2007.

[33] W. Bludau, A. Onton, and W. Heinke, "Temperature dependence of the band gap of silicon," Journal of Applied Physics, vol. 45, no. 4, pp. 1846-1848, 1974.

[34] C. Tserbak and G. Theodorou, "Energy band structure and linear optical properties of Si and Ge strained along the [111] and [110] directions," Physical Review B, vol. 52, no. 16, pp. 1223212240, 1995.

[35] M. Oshikiri, F. Aryasetiawan, Y. Imanaka, and G. Kido, "Quasiparticle effective-mass theory in semiconductors," Physical Review B, vol. 66, no. 12, Article ID 125204, 2002. 

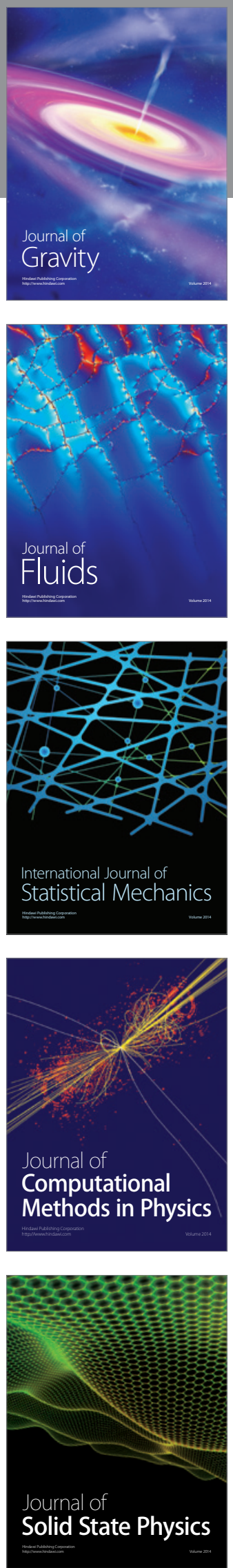

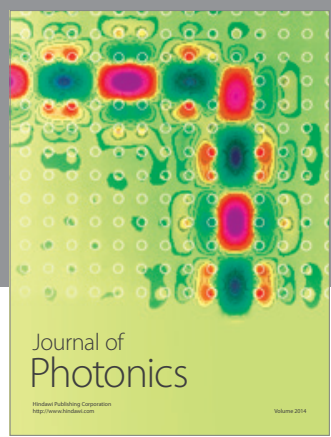

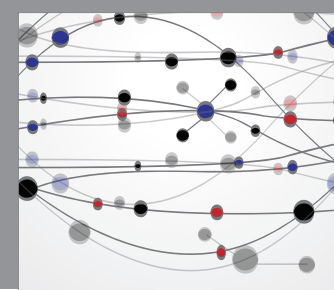

The Scientific World Journal

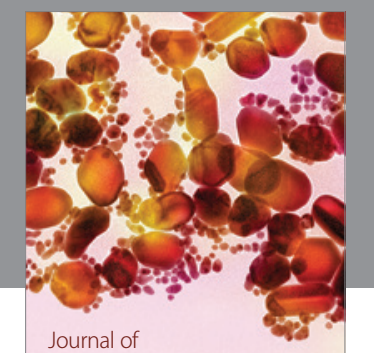

Soft Matter
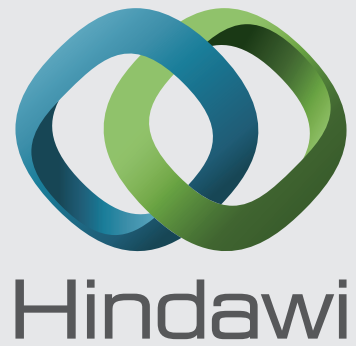

Submit your manuscripts at

http://www.hindawi.com
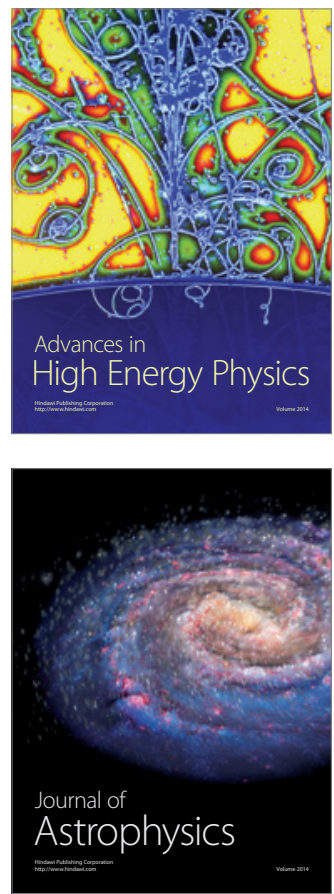
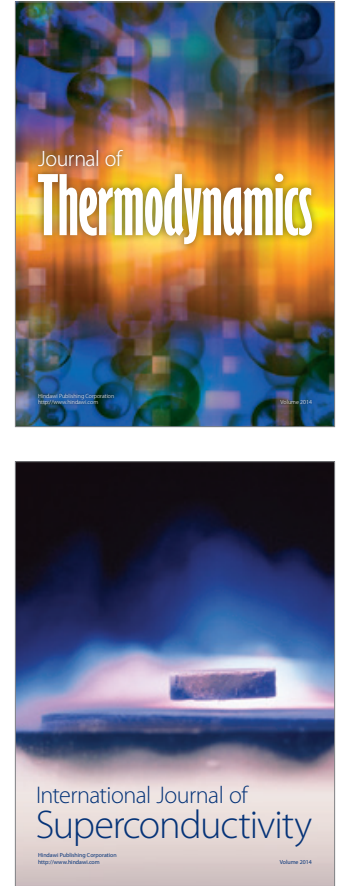
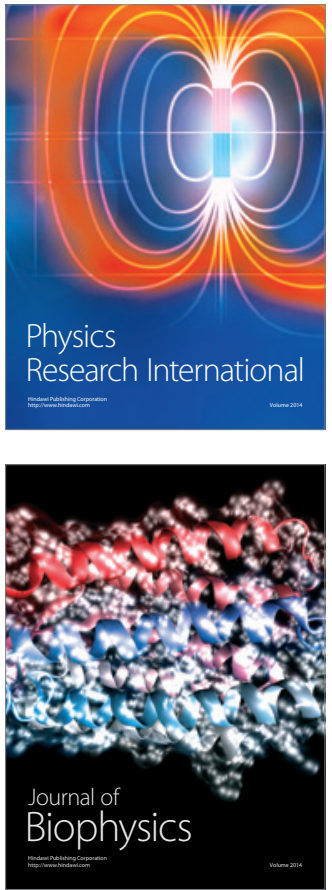
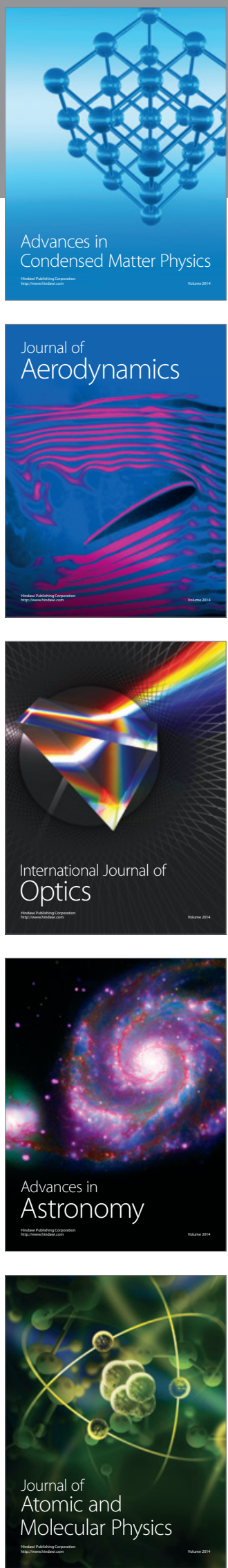